\title{
Beeinflusst die Einnahme von Statinen das Behandlungsergebnis der Strahlentherapie beim Prostatakarzinom?
}

\section{Originalpublikation}

Gutt R, Tonlaar N, Kunnavakkam R et al. Statin use and risk of prostate cancer recurrence in men treated with radiation therapy. J Clin Oncol 2010;28:2653-9.

Fragestellung:

In einer retrospektiven Analyse untersuchte die Arbeitsgruppe, ob die Einnahme von HMGCoA-Reduktase-Inhibotoren (Statine) zur Behandlung von Fettstoffwechselstörungen einen Effekt auf den Krankheitsverlauf eines Prostatakarzinompatienten nach Strahlentherapie (RT) hat [Gutt et al].

\section{Patienten und Methodik:}

691 Prostatakarzinom-Patienten mit bekannter Medikamentenanamnese wurden zwischen 1988 und 2006 einer definitiven RT unterzogen, und zwar perku$\tan (84 \%$; mediane Dosis 72 Gy), mit Brachytherapie (9\%; mediane Dosis 144 Gy) oder einer Kombination beider (7 \%). Pelvine Lymphknoten wurden in $7 \%$ der Fälle bestrahlt. Bei $41 \%$ kam eine Hormontherapie zum Einsatz. Zwei Subgruppen wurden bezüglich der biochemischen Rezidivfreiheit und des rezidivfreien Überlebens verglichen: Patienten, die während der Strahlentherapie oder danach eine StatinTherapie erhielten $(\mathrm{n}=189$, davon 150 Statine während der Radiotherapie und 39 Statine während der Nachbeobachtungszeit), und Patienten, die nie ein Statin erhielten $(n=502)$. Die eingesetzten Statine waren Atorvastatin,

Urologe 2013 $52: 264$

DOI 10.1007/s00120-013-3140-6

(c) Springer-Verlag

Berlin Heidelberg 2013
Lovastatin, Fluvastatin, Pravastatin, Simvastatin und Rosuvastatin. Patienten „mit Statinen“ wiesen signifikant niedrigere Ausgangs-PSA-Werte (Median 7,2 vs. $8,7 \mathrm{ng} / \mathrm{ml}$ ) und günstigere T-Stadien auf, waren häufiger mit IMRT behandelt worden (59 vs. $34 \%$ ), hatten eine höhere Gesamtdosis erhalten (median 74 vs. 72 Gy) und wurden häufiger antihormonell behandelt (47 vs. $39 \%$, statistischer Trend).

\section{Ergebnisse:}

Bezüglich des rezidivfreien Überlebens waren der PSA-Wert, die RT-Technik (Brachytherapie oder perkutane RT > 74 Gy), der Gleason-Score und die StatinEinnahme von signifikantem Einfluss. Die biochemische Rezidivfreiheit nach vier Jahren betrug mit Statinen $93 \%$ und ohne Statine $80 \%$, ohne Einfluss von Art und Dosierung. Patienten mit einem niedrigen Gesamtcholesterin oder niedrigem LDLCholesterin vor Beginn der RT erreichten eine längere biochemische Rezidivfreiheit. Multivariate Analysen deuten darauf hin, dass der LDL-Cholesterin-Plasmaspiegel und nicht die Statin-Therapie per se der dominierende prognostische Faktor ist.

\section{Schlussfolgerung:}

Prostatakarzinom-Patienten, die wegen einer Fettstoffwechselstörung während oder nach der RT mit Statinen behandelt werden, bleiben länger biochemisch rezidivfrei als ohne Statin-Einnahme. Es bleibt offen, ob dies auf die Statine zurückzuführen ist oder über den gesenkten Cholesterinspiegel vermittelt wird.

\section{Kommentar:}

In der Zusammenschau mit der bereits publizierten Literatur gibt die von Gutt et al. Vorgelegte Studie nur einen recht schwachen Hinweis auf die Möglichkeit, durch Statin-Einnahme eine Verbesserung der strahlentherapeu- tischen Ergebnisse des Prostatakarzinoms erreichen zu können. Die Modulation des Cholesterinstoffwechsels durch diese Substanzgruppe scheint die Biologie des Prostatakarzinoms zu beeinflussen, auch dürften Zusammenhänge mit anderen relevanten "lifestyle factors" bestehen. Eine tatsächliche Radiosensibilisierung der Prostatakarzinomzellen ist aus der aktuellen Datenlage für die Klinik nicht ableitbar.

Erstpublikation in: Strahlenther Onkol 2011;187(3):218-9

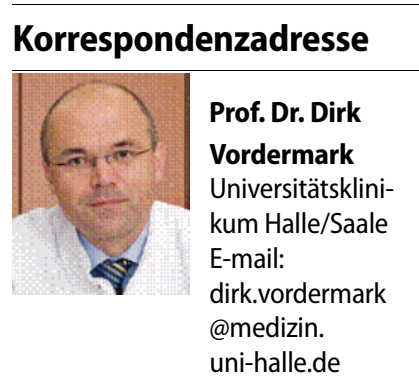

\section{Führt die Androgendeprivation bei Patienten mit Prostatakarzinom zu einer erhöhten kardiovaskulären Mortalität?}

\section{Originalpublikation}

Nguyen PL et al. Association of androgen de- de-privation therapy with cardiovascular death in patients with prostate cancer. JAMA 2011; 306: 2359-66

In einer Metaanalyse von acht prospektiv randomisierten Studien mit 4.141 Patienten wurde die sofortige GnRH-Agonistenbasierte ADT gegen keine oder verzögerte ADT bei Patienten mit nicht metastasiertem, aber prognostisch ungünstigem Prostatakarzinom untersucht.
Die Zahl kardiovaskulärer Todesfälle zwischen ADT-und Kontroll-Patienten unterschied sich (11,0 \% vs. $11,2 \%)$ nicht. Die Analyse von 4.805 Patienten aus elf Studien mit Daten zur Gesamtmortalität ergab, dass sofortiger Androgenentzug mit signifikant geringerer Prostatakarzinom-spezifischer $(13,5 \%$ vs. $22,1 \%)$ und Gesamtmortalität (37,7\% vs. $44,4 \%)$ einherging.

Die Metaanalyse fand somit keine Evidenz, dass Androgenentzug das Risiko für kardiovaskuläre Todesfälle bei Prosta-
takarzinom-Patienten steigert. Die Patienten profitierten jedoch beim Prostatakarzinom-spezifischen und Gesamtüberleben. Unklar bleibt, ob dies für Patienten mit chronischer Herzinsuffizienz oder nach Myokardinfarkt gilt.

\section{Ulrike Wepner}

Erstpublikation in: Uro-News 2012; 16 (3): 44 